\title{
The Central Temperature of the Stars
}

\author{
Angel Fierros Palacios \\ Instituto de Investigaciones Eléctricas, División de Energías Alternas, Mexico City, Mexico \\ Email: afierros@iie.org.mx \\ Received 4 June 2015; accepted 4 April 2016; published 8 April 2016 \\ Copyright (C) 2016 by author and Scientific Research Publishing Inc. \\ This work is licensed under the Creative Commons Attribution International License (CC BY). \\ http://creativecommons.org/licenses/by/4.0/ \\ (c) (i) Open Access
}

\section{Abstract \\ From the theory about the internal structure and stars stability, a relationship for the central temperature of any gaseous star can be obtained.}

Keywords

The Central Temperature of the Stars

\section{Introduction}

The value of the main parameters of the Sun and other stars, like the luminosity and the central temperature, can be obtained from the basic equations of the theory about the stability and equilibrium of the stars [1] [2]. However, given that some of its results are not totally satisfactory, it is necessary to modify that theory in order to get a new analytical scheme more wide and useful [1].

\section{The Self-Generated Magnetic Field and the Central Temperature}

Let us consider the following relations

$$
L=\frac{4 \pi G c M}{\alpha k_{c}}\left(\frac{1-\beta}{\beta}\right)
$$

This is the modified mass-luminosity relation [1]. Here, $L$ is the luminosity, $M$ the mass, $c$ the velocity of light in the empty space, $G$ the universal gravitational constant, $k_{c}$ the opacity coefficient at the center of the star, and $\alpha=2.5$ a constant [1] [2]. Moreover, $\beta$ is a parameter which represents the ratio between hot gases pressure and the whole pressure, while $1-\beta$ is the ratio between radiation pressure and the whole pressure [1] [2]. Then,

$$
\begin{aligned}
& (1-\beta) p=p_{r} \\
& \beta p=p_{g} ;
\end{aligned}
$$


where $p$ is the whole pressure, and

$$
\begin{aligned}
& p_{r}=\frac{1}{3} a T^{4} \\
& p_{g}=\frac{R \rho T}{\mu}
\end{aligned}
$$

are the radiation pressure and the hot gases pressure, respectively. In those relations, $T$ is the temperature, $\rho$ the mass density, $\mathbb{R}$ the gases universal constant, $\mu$ the average molecular weight, and $a=7.64 \times 10^{-15}$ the Stefan's constant [1] [2].

Now, from the momentum balance equation of magneto hydrodynamics [3], and for any gaseous star, it follows that [2] [3]

$$
H^{2}=\frac{4 \pi R \rho T}{\mu}
$$

where $H^{2}$ is the square of the intense magnetic field which all gaseous stars self-generate at an early stage of their evolution.

Substituting (2) and (3) in (4) we obtain that

$$
H^{2}=\frac{4 \pi a \beta T^{4}}{3(1-\beta)}
$$

and then

$$
T_{c}=\left[\frac{3 H^{2}}{4 \pi a}\left(\frac{1-\beta}{\beta}\right)\right]^{1 / 4}
$$

where the subscript $c$ means the temperature at the center of stars. However, $T_{c}$ and $H^{2}$ are directly related; so that another independent equation is necessary for the magnetic field. Hence, from the polytropic gas sphere theory [1]-[3], it can be obtained the relationship that follows

$$
H^{2}=2 \rho \pi \phi\left(\frac{1-\beta}{\beta}\right)
$$

where

$$
\phi=\frac{G M}{R}
$$

is the gravitational potential, $R$ the stellar radius, and $M$ the mass [1].

Substituting (7) and (8) in the relation (1) we have that

$$
H^{2}=\frac{\alpha k_{c} \rho_{c} L}{2 c R}
$$

Finally, with this result substituting in (6), it is easy to see that

$$
T_{c}=\left[\frac{3 \alpha k_{c} \rho_{c} L}{8 \pi c a R}\left(\frac{1-\beta}{\beta}\right)\right]^{1 / 4}
$$

Thus, for any gaseous star, the central temperature behaves as a constant, in the meantime, the power generating source can be feed with new nuclear fuel [1].

\section{Conclusions}

In the specialized literature [1]-[3], the values of central temperature of the Sun, and also its luminosity estimated with the use of the non modified theory about the stability and equilibrium of the stars [2], are over valuated. In fact, the data reported are the following 


$$
\begin{aligned}
& L_{\odot}=5.62 \times 10^{33} \mathrm{ergs} \cdot \mathrm{sec}^{-1} \\
& T_{c \odot}=39.5 \times 10^{6} \mathrm{~K} ;
\end{aligned}
$$

where the symbol $\odot$ indicates the Sun.

This is so, because the self-generated magnetic field has never been included in that theory [1] [2]. In order to get the before mentioned modification, that magnetic field was introduced in the fundamental equation that governed the state of equilibrium, which is now magneto-mechanical [1], in order to get more realistic values for those sun's basically parameters.

Hence, for $L_{\odot}$ the calculated and the observational data has the same value, while the central temperature has the following magnitude

$$
T_{c \odot}=16.5 \times 10^{6} \mathrm{~K} ;
$$

which is a more acceptable value, given the stability and the state of equilibrium of the Sun. In consequence, the relationship (10) is useful to calculate the central temperature for any gaseous star.

\section{References}

[1] Fierros Palacios, A. (2015) The Magnetic Field in the Stability of the Stars. Journal of High Energy Physics, Gravitation and Cosmology, 1, 88-113.

[2] Eddington, A.S. (1988) The Internal Constitution of the Stars. Cambridge University Press, Cambridge. http://dx.doi.org/10.1017/CBO9780511600005

[3] Fierros Palacios, A. (2006) The Hamilton-Type Principle in Fluid Dynamics. Fundamental and Applications to Magnetohydrodynamics, Thermodynamics, and Astrophysics. Springer, Wien. 